\title{
The IAU Office of Astronomy for Development
}

\author{
Kevin Govender \\ IAU Office of Astronomy for Development, P.O. Box 9, Observatory, 7935, South Africa \\ email: kg@astro4dev.org
}

\begin{abstract}
On 16 April 2011 the IAU's Office of Astronomy for Development (OAD) was launched jointly by the President of the IAU and the South African Minister of Science and Technology, at the South African Astronomical Observatory in Cape Town. This OAD was set up to realise the IAU's strategic plan which aims to use astronomy as a tool for development. Communicating astronomy with the public is one of the OAD's focus areas.
\end{abstract}

\section{Background}

The IAU adopted the strategic plan 2010-2020 at its 2009 General Assembly in Rio de Janeiro, Brazil. The basis of this strategic plan was to use astronomy to stimulate global development. With the slogan 'Exploring our Universe for the benefit of humankind' this plan aimed not only to stimulate the field of astronomy but also importantly to realize its benefits to humanity. The elements at the heart of the plan are primary, secondary and tertiary education, scientific research and public outreach. In order to realize this strategic plan the IAU established the Office of Astronomy for Development (OAD), which, after a lengthy international bidding process, was located in Cape Town, South Africa, at the South African Astronomical Observatory, and began operations in 2011.

\section{The Office of Astronomy for Development (OAD)}

The OAD is overseen by a steering committee made up of 3 nominations from the IAU and 3 nominations from the host organization in South Africa (the South African Astronomical Observatory, a facility of the National Research Foundation). Currently the steering committee consists entirely of IAU members and is led by George Miley. Although the OAD itself has only 3 full time staff, global expertise is sought in the form of its Task Forces. Task Forces are groups of international 'experts' in their fields who advise and assist the $\mathrm{OAD}$ in the implementation of the strategic plan. These are not necessarily IAU members but nominated and selected from a global science community.

Following an international stakeholder workshop in December 2011 and an open call for nominations, the three task forces were established. They are: (i) Astronomy for Universities and Research, led by a veteran of the IAU Commission 46, Edward Guinan; (ii) Astronomy for Children and Schools, led by the ex-IYA-coordinator, Pedro Russo; and (iii) Astronomy for the Public, led by one of the former presidents of IAU Commission 55 and current organising committee member, Ian Robson.

In addition to the Task Forces the OAD structure includes the establishment of regional nodes and language expertise centers. Regions covered by these nodes could be geographic (e.g. neighbouring countries) or cultural (e.g. globally distributed language or culture). This would allow for greater impact in that region and a more informed conversation with the IAU about particular local considerations when implementing the strategic plan in 
that region. Currently two regional nodes have been established: one in China for the East Asia region and mainly Chinese language and culture; and one in Thailand for the South East Asian region. Numerous negotiations are ongoing regarding the establishment of other nodes across the world.

The OADs vision 'Astronomy for a better world' captures the essence of the IAU strategic plan and allows the freedom to innovate in terms of the impact that astronomy can have on society. The OAD as such aims to further the use of astronomy as a tool for development by mobilizing the human and financial resources necessary in order to realize its scientific, technological and cultural benefits to society. The OAD has embarked on a recruitment drive to find volunteers willing to contribute to the implementation of the strategic plan. To date over 400 volunteers have signed up via the OAD website listing their skills and potential contributions. These volunteers are called upon as specific opportunities arise. They are also encouraged to participate in the OAD calls for project proposals so that their ideas can be supported.

In August 2012 the first open call for proposals was released with the IAU allocating funds towards projects relating to each of the Task Forces. The principle of the call was that each Task Force would evaluate proposals received and decide which were to be funded and which were to be put onto a wish list - the OAD would then continue to fundraise for those wish list projects. Under Task Force 3 (Astronomy for the Public) proposals were sought for activities such as journalist/blogger/communicator and amateur astronomer training; citizen science projects; models for outreach activities; traditional/cultural astronomy; databases of existing celebrations; creation of images and multimedia resources; stargazing events; astro-tourism activities; outreach awards; evaluation guidelines; etc.

In order to support the anticipated demand for projects the OAD has already established relationships with several organizations including the Royal Astronomical Society, the Netherlands Organisation for Scientific Research, the International Science Programme (Uppsala University), the International Centre for Theoretical Physics, the Inter-University Centre for Astronomy and Astrophysics and the University of Central Lancashire.

\section{Public Outreach within the IAU}

The OADs Task Force 3 (Astronomy for the Public), is certainly not the only activity of the IAU in terms of Public Outreach, nor should it be, given the wide reaching nature of the field and the many activities from IYA that need to be sustained. The IAU has also established the Office for Public Outreach which is based at the National Astronomical Observatory of Japan. This office is led by the Public Outreach Coordinator (Sarah Reed) and its purpose is to 'Organize and conduct public outreach activities of the IAU by coordinating and sharing responsibilities with the IAU Office of Astronomy for Development.'

In addition to this office the IAU still has within its structures Commission 55 (Communicating Astronomy with the Public). This Commission has been responsible for the very successful series of Communicating Astronomy with the Public (CAP) conferences as well as the publication of the CAP Journal, amongst its other activities such as the Washington Charter and the Virtual Astronomy Multimedia Project. The work of this Commission will thus certainly still be needed in the mix of IAU public outreach. In fact it will be essential that all three structures (the OAD Task Force 3, the Office of Public Outreach, and IAU Commission 55) work closely together and synergise for effective communication of astronomy with the public. 